\title{
Stacking of Interferometric Data
}

\author{
Lukas Lindroos ${ }^{1}$ and Kirsten K. Knudsen ${ }^{1}$ \\ ${ }^{1}$ Department of Earth and Space Sciences, \\ Chalmers University of Technology, Onsala Space Observatory, 43992 Onsala, Sweden \\ email: lindroos@chalmers.se, kraiberg@chalmers.se
}

\begin{abstract}
Radio and mm observations play an important role in determining the star formation properties of high redshift galaxies. However, most galaxies at high redshift are too faint to be detected individually at these wavelengths. A way to study this population of galaxies is to use stacking. By averaging the emission of a large number of galaxies detected in optical or near infrared surveys, we can achieve statistical detection.

We investigate methods for stacking data from interferometric surveys. Interferometry poses unique challenges in stacking due to the nature of imaging of this data. We have compared directly stacking the $u v$ data with stacking of the imaged data, the latter being the typically used approach. Using simulated data, we find that $u v$-stacking may provide around $50 \%$ less noise and that image based stacking systematically loses around $10 \%$ of the flux.
\end{abstract}

Keywords. Stacking, Interferometry

\section{Stacking algorithms and comparison based on simulated data}

The aim of our work was to investigate how to best stack data from interferometric surveys. We have evaluated two algorithms. One method based on fully imaged data, similar to the method used by Carilli et al. (2008) and one method working directly on the visibility data.

To evaluate the algorithms, we produced a simulated data set with 100 sources at 25 mJy. In order to create a more realistic noise, several brighter sources around a few Jansky were also introduced. Using this data, the image stacking algorithm yielded a 1.5 times higher noise level and systematically missed $10 \%$ of the flux. Stacking the $u v$-plane data reproduces the expected result well within the error bars.
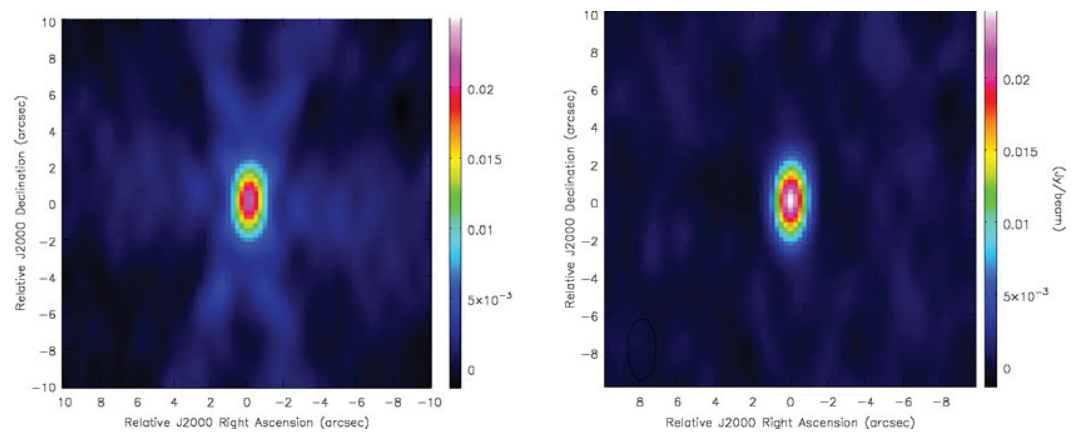

Figure 1. Comparison of stacking done on the image-plane (left) and the uv-plane (right).

\section{References}

Carilli, C. L., et al. 2008, ApJ, 689, 883 